\title{
THE INFLUENCE OF THE RADIATION SOURCE PARAMETERS ON THE ACCURACY OF DIGITAL SPECKLE CORRELATION METHOD
}

\author{
Lin $\mathrm{Li}^{1 *}$, Fedor Gubarev ${ }^{1,2}$ \\ ${ }^{1}$ National Research Tomsk Polytechnic University, 634050, Tomsk, Russia \\ ${ }^{2}$ V.E. Zuev Institute of Atmospheric Optics, Russian Academy of Sciences, Siberian Branch, 634050, \\ Tomsk, Russia
}

\begin{abstract}
The article presents the impact of the laser radiation divergence and the wavelength on the harmonic distortion detection, as well as the influence of the distance between the optical circuit elements on the harmonic distortion and the sensitivity of amplitude. The research was made by recording the speckle images from the vibrating surface of the object with its digital processing later.
\end{abstract}

\section{Introduction}

The method of digital speckle correlation is one of the most common methods for optical remote control and monitoring. The development of nano- and micro-technologies of modern systems brings to the fore the need to study in details this method to obtain the information about objects and processes. As objects of observation can serve machine parts, any mechanisms or living organisms, the sphere of its application is very broad [1-5]. This technique makes possible to receive the pattern, carrying information about the dynamics of the object, by the application of digital image processing systems, comparing the speckle images of the object surface. The method of digital speckle correlation also holds promise for diagnostics in biology and medicine, as it took a significant place in the non-destructive testing of materials [6-9].

The authors [10] consider a number of advantages of this method by comparing it with other methods, in particular with the Doppler method. In their work they also consider the ways of non-contact blood pressure measurement, using digital speckle correlation.

The optical monitoring revealed an interesting feature of the method; it is its ability to read information using the object vibration. The vibration in some processes presents vital information (voice, heartbeat, blood circulation, breathing, etc.), the research in this area presents interest from the point of view of remote monitoring.

The authors [11] examined the optical system, which allows to extract information from vibrating objects located at the distance of $100 \mathrm{~m}$ using the method of digital speckle correlation, which demonstrates a great potential of this method. A number of other studies [12-20] also demonstrate the ability to read the information in this way. Nevertheless, the

*Corresponding author: wojiaolilin@mail.ru 
problem affecting the quality of the information obtained by this method is not sufficiently investigated.

Therefore, the aim of this work is to study the distortion during the process of decoding the optical signals received by the digital speckle correlation.

\section{Experimental techniques}

The test object was a cone speaker of the active computer speakers, which are fed from a digital harmonic signal generator SFG-72120 (GW Instek). The diameter of the cone speaker was $7 \mathrm{~cm}$. The voltage amplitude of the loudspeaker was measured by a digital oscilloscope Rigol DS1052E. Unfortunately, we do not know the characteristics of that speaker system (frequency response, phase response), and how well the speaker converts the electrical oscillations into the mechanical ones. But, since we apply the sinusoidal voltage to the speaker, it can be assumed that the oscillation of the speaker cone will also be sinusoidal, as the harmonic oscillations are elementary.

The formation of speckle patterns is carried out by a $5 \mathrm{~mW}$ He-Ne laser $(632 \mathrm{~nm})$ and a $5 \mathrm{~mW}$ semiconductor laser $(532 \mathrm{~nm})$ used as a source of a coherent radiation. The beam diameter and the divergence of the He-Ne laser and of the semiconductor laser are $0.8 \mathrm{~mm}$, $1.2 \mathrm{~mm}$ and $3 \mathrm{mrad}, 2 \mathrm{mrad}$, respectively. The laser beam was directed to the cone speaker. Speckle patterns were recorded using a high-speed camera Fastec CMOS HiSpec 1 with the lens F0.95 Navitar. The camera was placed at the distance of $50 \mathrm{~cm}$ from the loudspeaker. Recorded images were transferred to a computer for further processing. The description of the speckle patterns processing method and the results of its testing are given in [21].

The procedure of investigation, used in this study, is shown in Figure 1. The scheme is used to study the dependence of the quality of the information read from the vibrating object surface. The studies were conducted under various conditions: the diameter of the spot on the surface of the object was varied by changing the distance between the object and the lens; two different radiation sources were used; lenses with different focal lengths, which provided the illumination of the object by the light with a different divergence, were used.

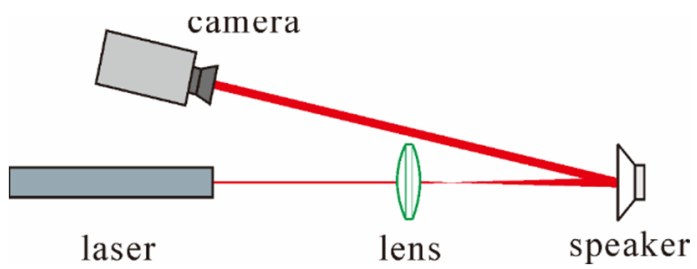

Figure 1. Schematic illustration of the experiment.

\section{Results}

Due to the fact that the speckle patterns are recorded by a digital camera, i.e. digitized, it can, under certain conditions, make a significant error. Thus, when the speckle size is of the order of the pixel size of the camera sensor or less, the recorded speckle pattern will have a low resolution, which leads to deterioration of the accuracy of oscillation surface decoding. In the case when the size of the speckle is many orders greater than the pixel size, the resolution of the recorded speckle pattern will, of course, be high, however, the quantity of information, contained in the speckle pattern of the same size, will be much less. Therefore, the latter case requires the increase of the speckle pattern size, which leads to the increase of the processing time. 
It is well known [22] that the speckle size is directly proportional to the distance between the subject and the camera, and is inversely proportional to the diameter of the laser beam. Figure 2 shows the speckle pattern obtained with different spot size of the laser radiation on the speaker cones. The presented images can demonstrate that by increasing the diameter of the laser spot size the speckle is reduced. The regulation of the laser spot diameter is achieved by moving the focusing lens along the optical axis. However, it should be noted that while the divergence of the laser beam is increased (for the focusing lens, used in the experiment, the divergence of laser radiation was $11 \mathrm{mrad}$ ). The choice of such a scheme of the experiment is due to the parallel study of the possibility to apply the simplest scheme of the laser beam diameter varying to detect the objects surface oscillation.

In our next experiment we studied the effect of the laser spot diameter of the object surface on the quality of the process of detection its oscillations. Figure 3 shows the dependence of the sensitivity fluctuation of the amplitude of the decoded signal of the distance between the focusing lens and the speaker cone. The focal distance of the lens was $11 \mathrm{~cm}$. The Figure 3 shows that the movement of the focusing lens from the laser to the speaker leads, firstly, to the decrease of the sensitivity and then to its increase, secondly. The dependence of the sensitivity is expressed in the minimum range distance of 20 to 30 $\mathrm{cm}$ (Figure 3). The minimum value of sensitivity in the experiments was about $1 \mathrm{pixel} / \mathrm{V}$.

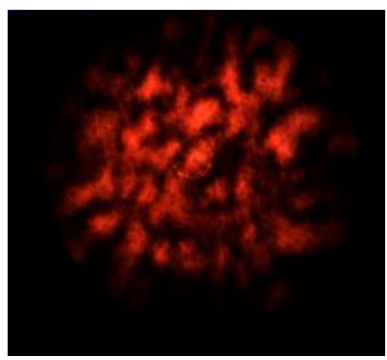

$\mathrm{a}$

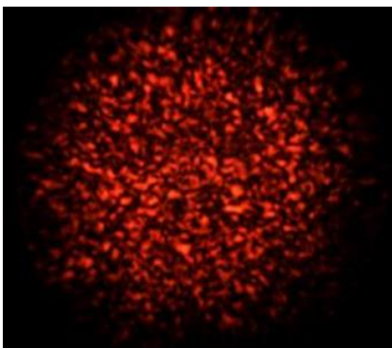

b

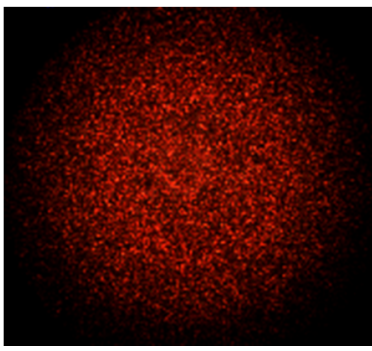

c

Figure 2. The speckle patterns with different diameter laser spot on the diffuser speaker: a) $11 \mathrm{~mm}, \mathrm{~b}$ ) $17 \mathrm{~mm}$, c) $47 \mathrm{~mm}$. The distance between the surfaces of the object and the camera is $50 \mathrm{~cm}$. The camera's exposure time is $1995 \mathrm{~ms}$.

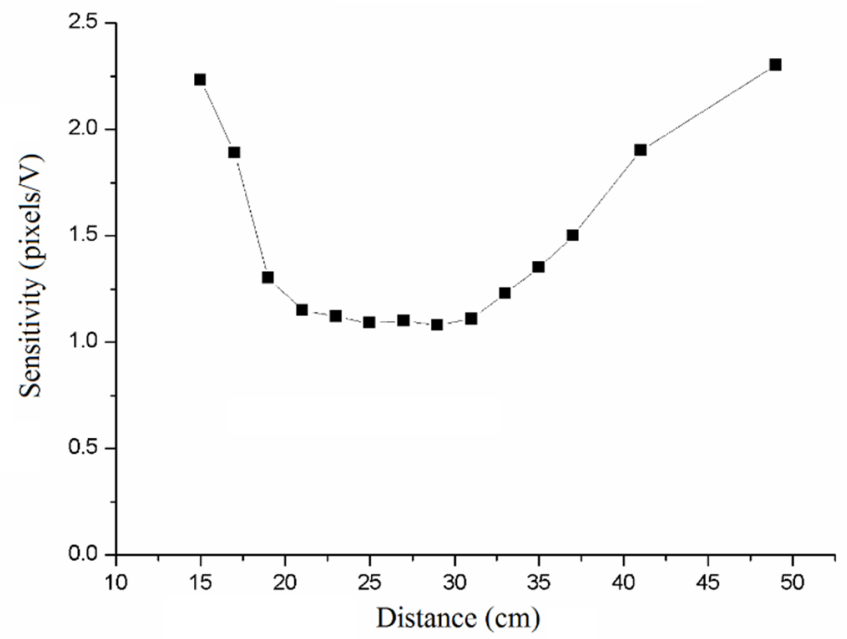

Figure 3. The dependence of sensitivity fluctuation amplitude of the decoded signal of the distance between the focusing lens and the speaker cone. The signal frequency is $50 \mathrm{~Hz}$. The frequency of camera registration is $500 \mathrm{~Hz}$, the camera's exposure time is $1.995 \mathrm{~ms}$. 
It should be noted that the speckle size influences on the optimum size of the image area used for speckle tracking shift. Since the displacement of the lens changes the size of speckles (Figure 1), it is necessary each time to select the optimal size of the image area while moving the lens. The search for optimal image speckle size was based on minimizing the value of the total harmonic distortion (THD) of the decoded signals.

The study also obtained the dependence of THD decoded signal on the sinusoidal voltage amplitude signal applied to the speaker coil, at various distances between the loudspeaker and the focusing lens. The dependence was deducted by averaging the experimental data. The dependence of the THD decoded signal average value on the distance between the focusing lens and the loudspeaker is shown in Figure 4. In addition to the fact that reducing the distance to values close to the focal distance of the lens, led to the sensitivity increase (Figure 3), it also led to a significant increase of the decoded signal THD value (Figure 4). By varying the distance ranging from $15 \mathrm{~cm}$ to 47 the THD decoded signal varied in the range from $5 \%$ to $15 \%$.

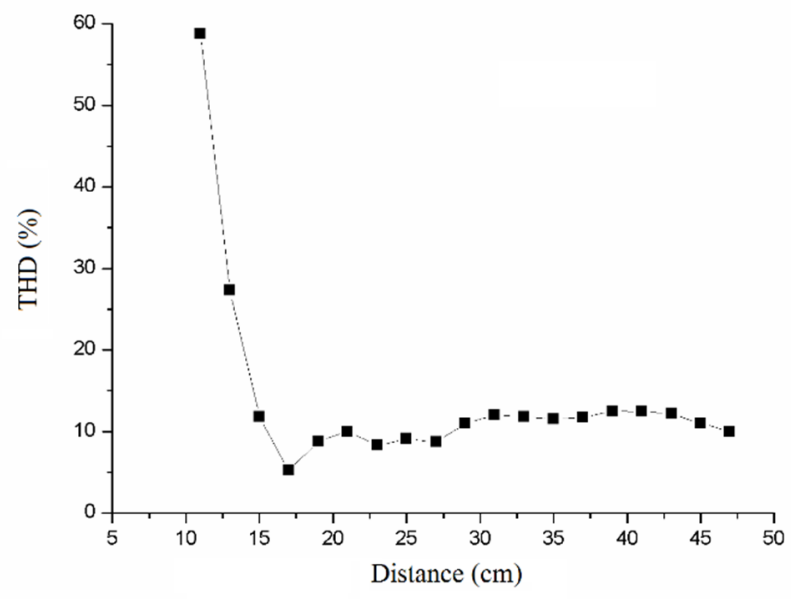

Figure 4. Dependence of the THD decoded signal average value on the distance between the focusing lens and the loudspeaker. The frequency of the sinusoidal signal is $50 \mathrm{~Hz}$. The frequency of camera recording is $500 \mathrm{~Hz}$, the camera's exposure time is $1.995 \mathrm{~ms}$.

In addition to the study of the influence of the spot diameter on the quality of the received information, this paper studied the effect of beam divergence on the quality of information. The circuit shown in Figure 1 was also used to change the divergence through the use of different lenses and two different radiation sources: He-Ne laser $(632 \mathrm{~nm})$ and green semiconductor laser $(532 \mathrm{~nm})$. Figure 5 presents the speckle patterns, received by using different light sources. In this series of experiments the He-Ne laser was used without lens and with lenses of different focal length: $8 \mathrm{~cm}, 11 \mathrm{~cm}$ and $50 \mathrm{~cm}$. Figure 6 shows the average value of the THD decoded signal on the beam divergence value using the He-Ne laser. One can see that with the increase of divergence value, the THD value also increases. 


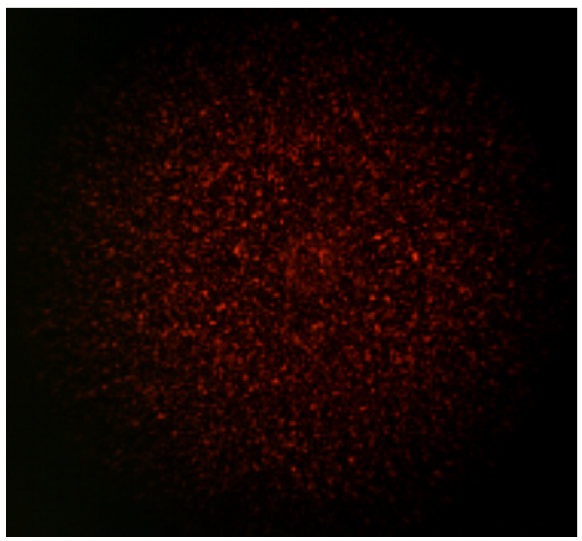

a

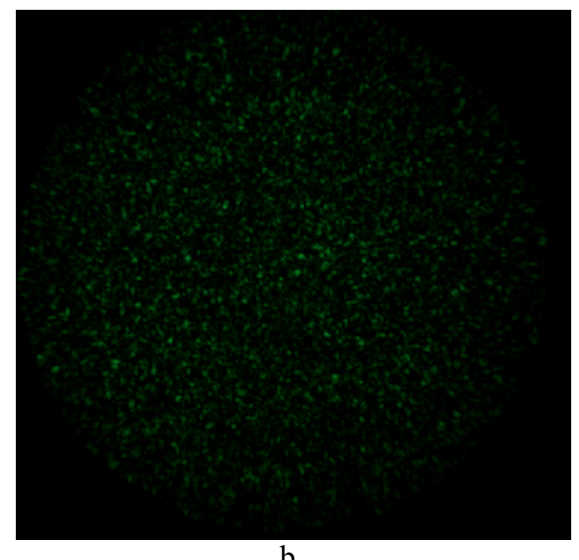

b

Figure 5. The speckle pattern with different sources of radiation: a) He-Ne laser (632 nm), b) a green semiconductor laser $(532 \mathrm{~nm})$. The distance between the surfaces of the object and the camera is 50 $\mathrm{cm}$. The camera's exposure time is $1.995 \mathrm{~ms}$.

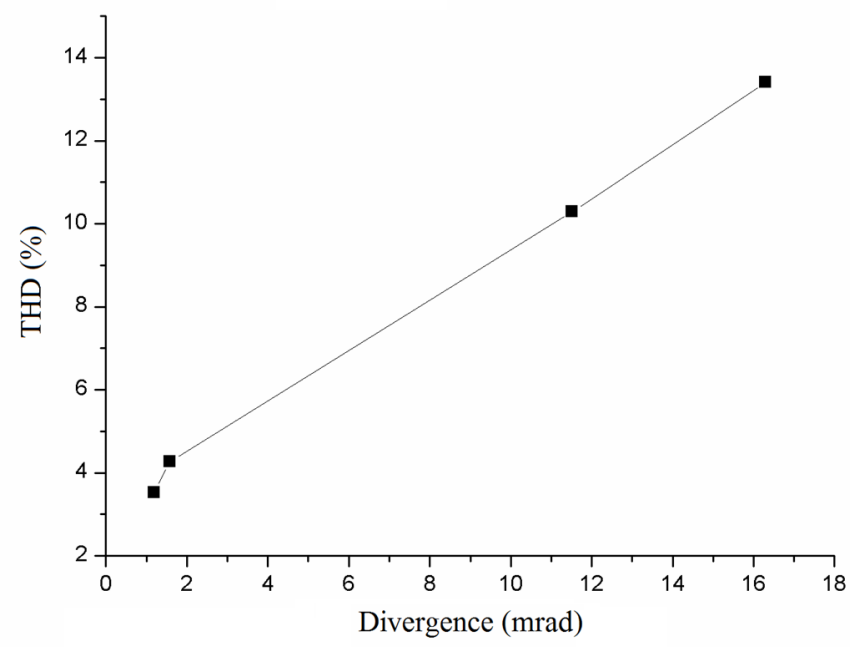

Figure 6. The dependence of the decoded signal average value on the THD values of the beam divergence using the He-Ne laser. The amplitude of the signal voltage is $2 \mathrm{~V}$. The signal frequency is $50 \mathrm{~Hz}$. The frequency of camera registration is $500 \mathrm{~Hz}$, The camera's exposure time is $1.995 \mathrm{~ms}$. 


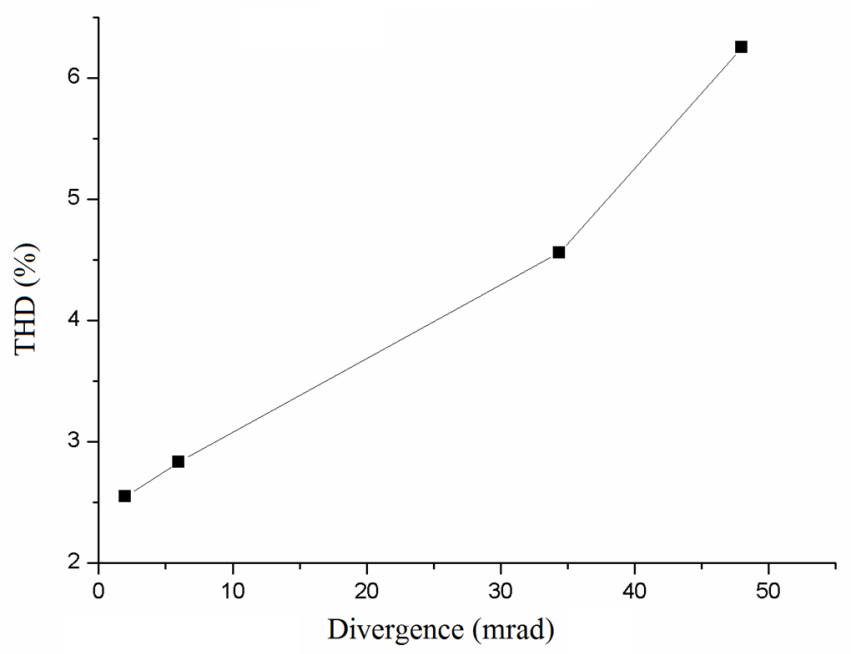

Figure 7. The dependence of the decoded signal average value on the THD value of the beam divergence using the green semiconductor laser. The amplitude of the signal voltage is $2 \mathrm{~V}$. The signal frequency is $50 \mathrm{~Hz}$. The camera registration frequency is $500 \mathrm{~Hz}$. The camera's exposure time is 1.995 ms.

In the next series of experiments the green semiconductor laser was used without a lens and with lens of different focal length: $8 \mathrm{~cm}, 11 \mathrm{~cm}$ and $50 \mathrm{~cm}$, as in the previous experiments. Figure 7 shows the dependence of THD decoded signal average value on the value of beam divergence when using the green semiconductor laser. It also shows that with increasing of divergence values the THD value also increases. However, this value is much smaller if we compare it with the THD average value when using He-Ne laser.

\section{Conclusions}

In this paper we studied the quality of data acquisition from a vibrating object surface by means of a digital speckle correlation method. The vibration of loudspeaker was used, to which was applied a sinusoidal voltage as the test object. The quality of the information extracting was assessed by comparing the decoded signals with the harmonic ones.

It is shown that the divergence of the beam affects the received information quality. The smaller the divergence of the beam is, the smaller the value of the coefficient of harmonic distortion is, i.e. it provides the better information extraction.

Using a collecting lens to illuminate the object, when the distance between the controlled object and the lens reduces to values, which are close to the focal distance of the lens, on the one hand, it leads to an increase of sensitivity, on the other, to a significant increase of the harmonic distortion values of the decoded signal. Thus by varying the distance between 15 to $47 \mathrm{~cm}$ the total harmonic distortion of the decoded signal was changing in the range from $5 \%$ to $15 \%$ under the experimental conditions.

\section{References}

[1] S. Noh, I. Yamaguchi, Jpn. J. Appl. Phys. 31, L1299 (1992) doi: 10.1143/JJAP.31.L1299 
[2] D.J. Chen, F.P. Chiang, Y.S. Tan, H.S. Don, Appl. Opt. 32, 1839 (1993) doi: 10.1364/AO.32.001839

[3] T. Fricke-Begemann, Appl. Opt. 42, 6783(2003)

[4] D. Amodio, G.B. Broggiato, F. Campana, G.M. Newaz, Experimental Mechanics 43,396 (2003) doi: 10.1177/0014485103434004

[5] J. Guanchang, W. Zhen, B. Nikeng, Y. Xuefeng, Opt. and Lasers in Engineering 39, 457 (2003) doi: 10.1016/S0143-8166(02)00028-3

[6] B.R. Durig, W.H. Peters, M.A. Hammer, Proc. SPIE. 0954, 438 (1989)

[7] V.V. Tuchin, J. Biomed. Opt. 4, 106 (1999)

[8] M.A. Vilensky, D.N. Agafonov, D.A. Zimnyakov, V.V. Tuchin, R.A. Zdrazhevskii, Quantum Electron 41, 324 (2011)

[9] M. Golberg, D. Fixler, A. Shainberg, et al, J. Biomed. Opt. 18, 101310 (2013)

[10] Y. Beiderman, I. Horovitz, N. Burshtein et al, J. Biomed. Opt. 15, 061707 (2010) doi: $10.1117 / 1.3505008$

[11] Z. Zalevsky, Y. Beiderman, I. Margalit, S. Gingold, M. Teicher, V. Mico, J. Garcia, Opt. Express 17, 21566 (2009) doi: 10.1364/OE.17.021566

[12] W.O. Wong, Opt. and Lasers in Engineering. 28, 277 (1997)

[13] Sama Molaie, Sohrab Ahmadi, Hadi Seyed Arabi, Indian J. Sci. Res. 3, 90 (2014)

[14] S. Bianchi, Appl. Opt. 53, 931 (2014) doi: 10.1364/AO.53.000931

[15] Z. Chen, C. Wang, C. Huang, H. Fu, H. Luo, H. Wang, Opt. Communications 331, 6 (2014) doi: 10.1016/j.optcom.2014.05.038

[16] Z. Chen, J. Chen, Journal of Vibration and Shock 34, 121 (2015)

[17] J. Quisberth, Z. Wang, H. Nguyen, Conference Proceedings of the Society for Experimental Mechanics Series 9, 105 (2016) doi: 10.1007/978-3-319-21765-9 14

[18] P. Vaz, T. Pereira, E. Figueiras, C. Correia, A. Humeau-Heurtier, J. Cardoso, Biomedical Signal Processing and Control. 25, 188 (2016) doi: 10.1016/j.bspc.2015.11.013

[19] C. Lal, A. Banerjee, N.U. Sujatha, J. Biomed. Opt. 18, 111419 (2013) doi: 10.1117/1.JBO.18.11.111419

[20] N. Sujatha; A. Banerjee, Proc. SPIE. 9302, 93021R (2015)

[21] F.A. Gubarev, L. Li, M.S. Klenovskii, A.Ph. Glotov, MATEC Web of Conferences 48, 04003 (2016) doi: 10.1051/confmatec/20164804003

[22] M. Franson, Speckle optics (Mir, Moscow, 1980) 